\title{
Two-dimensional Few-circle Optical Pulses of Bessel Profile in Bragg- environment with Carbon Nanotubes
}

\author{
M.B. Belonenko ${ }^{1,2}$, I.S. Dvuzhilov"1,*,Yu.V. Nevzorova ${ }^{1}$, O.Yu. Tuzalina ${ }^{3}$ \\ 1 Volgograd State University, 400062 Volgograd, Russia \\ ${ }^{2}$ Laboratory of Nanotechnology, Volgograd Institute of Business, 400048 Volgograd, Russia \\ 3 Volgograd State Agricultural University, 400002 Volgograd, Russia
}

(Received 25 April 2016; published online 03 October 2016)

\begin{abstract}
We consider the task about few-circle optical pulses dynamics (light bullet) with Bessel profile in the environment with spatial modulate refractive index with carbon nanotubes. We show that light bullets propagate stably. Electromagnetic field of pulse describes classically, on basis of Maxwell equation, and carbon nanotubes give dispersion law for electrons, which interacting with pulse. We show that light bullets propagate stably.
\end{abstract}

Keywords: Few-circle optical pulses, Bragg environment, Bessel beam, Carbon nanotube.

DOI: $10.21272 /$ jnep.8(3).03042

PACS numbers: 72.20.Ht, 42.65.Re

\section{INTORDUCTION}

At last some years we can see a huge interest in the researches of new types so-called light bullets [1-2]. We need to mark that light bullets present an electric field pulse, localized in the spatia an $d$ all energy of this pulse focus in the final limited area of spatial.

A new type of the optical pulse became popular among varieties of light beams - optical pulse with Bessel profile. Its own attention among theorist and experimenters it get at the expense of unique characteristic - ray likeable, it propagates without refraction and dissipation. Also, in theory, it transfers an infinite power, that allow to realize it practically. Though, at the reasonable good approximation it get its own application in optics, so the diffraction is absent.

The greate interst to this pulse very wide spectrum of the practical application, such as ultrahigh resolution microscopy, the communication activity by optical fiber communication lines, manipulations with micro- and nanoparticles and etc. [3-6].

Widely usefull in applications [7-9] CNT have a brightly expressed non-linear property and can be used as an environment, where light bullets are generating.

The velocity of optical beam propagation defines by environment reflective index only, where it propagate and can't change to wide limits.

Exit from that position is known: we need additionally to modulate the environment refractive index by any way, to make the Bragg lattice or environment.

In that case the velocity of wave packet propagation, as a result of it partial reflection and next nterference, will be defines by period and modulation depth of the refractive index. Than theoretically we can manage by the light beam velocity in such environment. Practically, the reflective index modulation is possible with help of the external constant field for environment with CNT, which have the Kerr effect. The importance of practical applicatios and our articulate consideration were stimulus for the writing that article.

\section{PRINCIPAL EQUATIONS}

The research of the CNT electron structure, as rule, strong coupling approximation within the bounds of analysis of $\pi$-electron dynamics. The dispersion low for CNT zig-zgg type $(m, 0)$ has a view[10]:

$$
\varepsilon_{s}(p)= \pm \gamma \sqrt{1+4 \cos (a p) \cos (\pi s / m)+4 \cos ^{2}(\pi s / m)}(1)
$$

Where $\gamma=2,7 \mathrm{eV}, \quad a=3 b / 2 \hbar, \quad b=0.142 \mathrm{~nm}-\mathrm{a}$ distance between neighbouring carbon atoms, and quasi-momentum $p z$ we can write as $(p z, s), s=1,2 \ldots m$.

At our construction the propagation model of the Bessel optical beam in Bragg environment subject to CNT system, in case of geometry presents at Fig.1., we will describe the electromagnetic field of the pulse by the Maxwell equations in the Coulombs gauge [11] $\mathbf{E}=-\partial \mathbf{A} / c \partial t$. Vector-potential has

$$
\mathrm{a} \frac{\partial^{2} \boldsymbol{A}}{\partial x^{2}}+\frac{\partial^{2} \boldsymbol{A}}{\partial y^{2}}-\frac{n^{2}(x, y)}{c^{2}} \frac{\partial^{2} \boldsymbol{A}}{\partial t^{2}}+\frac{4 \pi}{c} \boldsymbol{j}=0
$$

Where $n(x, y)$ give a spatial change of the reflective index, that is Bragg- lattice, $\mathbf{j}$-current, as a result of influence by electric field of pulse to electrons in the conduction band CNT. Here we don't take into account a diffraction spreading of the laser beam in the direction along CNT axis. Secondly, we don't take into account a electric field of the bottom layer.

Within the bounds of our model, we don't take into account interband transitions, its limit a laser pulse frequency, which lies in near infrared area.

We need to mark, a typical size of the CNT and distance between nanotubes more smaller that typical size of the space area, where localized a few-circle optical pulse, then we can use the continuous system approximation. And the current distributes by volume.

*Dvuzhilov.Ilya@gmail.com 


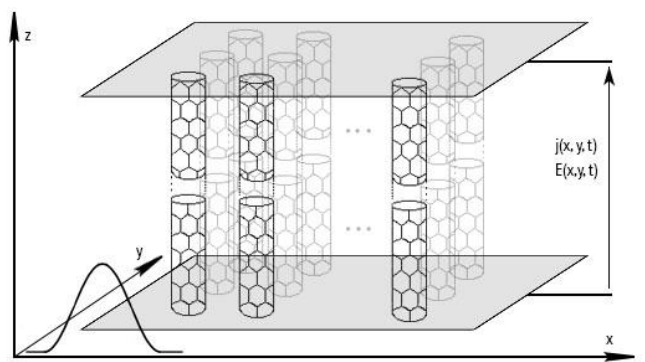

Fig. 1 - Task geometry, where $j(x, y, t)$ is a current along CNT axis, $E(x, y, t)$ is a pulse electric field

The typical length, where the reflective index changes appreciably for Bragg environment is more bigger and doesn't bring in supplementary limitations.

As far as typical relaxation time for electronsin CNT we can fix as $10^{-3} \mathrm{~s}$ [10], than electrons ensemble on typical times for tasks of the few-circle optical pulse dynamics (order of $10^{-14} \mathrm{~s}$ ) we can describe with help of kinetic Boltzmann equation [11]:

$$
\frac{\partial f}{\partial t}-\frac{q}{c} \frac{\partial \mathbf{A}_{z}}{\partial t} \frac{\partial f}{\partial p}=0
$$

Here $f=f\left(p_{s}, s, t\right)$ is a distribution function, which indirectly depends on coordinate (by act of law of dependence on vector-potential coordinate $\mathbf{A}$ ), and a distribution function $f$ at the initial moment of time match with function $F_{0}$ - equilibrium distribution Fermi function

$$
F_{0}=\frac{1}{1+\exp \left\{E(\mathbf{p}) / k_{b} T\right\}}
$$

Here $T$ - temperature, $k_{b}$ - Boltzmann constant.

For current density $\mathbf{j}=\left(0,0, j_{z}\right)$ :

$$
j_{z}=\frac{q}{\pi \hbar} \sum_{s} \int d p_{z} v_{z} f
$$

Here brings group electron velocity $v_{z}=\partial E(\mathbf{p}) / \partial p_{z}$, we solved equation (3) by method of characteristics [12], we can get:

$$
j_{z}=\frac{q}{\pi \hbar} \sum_{s} \int_{-q_{0}}^{q_{0}} d p_{z} v_{z}\left[p-\frac{q}{c} A_{z}(t)\right] F_{0}(\mathbf{p})
$$

Integration in (5) first we do by the first Brillouin zone and $q_{0}=2 \pi \hbar / 3 b$. Group velocity subject to dispersion low we can factorized to Fourier series:

$$
v_{z}(s, x)=\sum_{m} a_{m s} \sin (m x)
$$

here

$$
a_{m s}=\frac{1}{\pi} \int_{-\pi}^{\pi} v_{z}(s, x) \sin (m x) d x,
$$

expansion

coefficients, decrease with $m$ growth substitute: Finally, the effective equation can be present in a view [12]:

$$
\frac{\partial^{2} A_{z}}{\partial x^{2}}+\frac{\partial^{2} A_{z}}{\partial y^{2}}-\frac{n^{2}(x)}{c^{2}} \frac{\partial^{2} A_{z}}{\partial t^{2}}+\frac{q}{\pi \hbar} \sum_{m} c_{m} \sin \left(\frac{m a q}{c} A_{z}(t)\right)=0
$$

$$
c_{m}=\sum_{m} a_{m s} b_{m s}, \quad b_{m s}=\int_{-q_{0}}^{q_{0}} d p_{z} \cos \left(m a p_{z}\right) F_{0}(\mathbf{p})
$$

If with $m$ growth the coefficients $c_{m}$ decrease, than in sum (5), for calculations, we can restrict by the first tens of summands, and increase in further summand numbers, depends on requests to accounts precision.

\section{NUMERICAL ACCOUNT}

Equation (5) were solved numerically by using a direct finite-difference cross-like scheme [13]. Stride by time and coordinate are determine by standard conditions of stability, even so, strides of finitedifference scheme are halved serially, until the solution didn't change in 8th sign. The initial condition for vector-potential of the electric field of the light bullet was chose as a zero-order Bessel function of the first kind:

The initial condition for vector-potential of the light bullet electric field choose in Gaussian form:

$$
\begin{gathered}
A(x, y, 0)=Q \exp \left(-\frac{\left(x-x_{0}\right)^{2}}{\gamma_{x}}\right)\left(-\frac{\left(y-y_{0}\right)^{2}}{\gamma_{y}}\right) \\
\frac{d A(x, y, 0)}{d t}=Q \exp \left(-\frac{\left(x-x_{0}\right)^{2}}{\gamma_{x}}\right) J_{0}\left(\left|\frac{y-y_{0}}{\gamma_{x}}\right|\right) \exp \left(-\Delta\left|y-y_{0}\right|\right)
\end{gathered}
$$

Here $Q$ - the pulse amplitude, $\Delta$ - cutting parameter, which brings, because the beam with Bessel profile physically impossible and we need to cut the function to big distances for receipting of the final activation energy. $\gamma_{x}-$ pulse width to $\mathrm{X}$ axes, $\gamma_{y}-$ Bessel function parameter, $v$ - initial pulse velocity. Reflective index of the environment was modulated as:

$$
n(x)=n_{0}(1+\alpha \cos (2 \pi x / \chi))
$$

Where $\alpha$-modulation depth of the reflective index, $x-$ Bragg lattice period.

As shown results of our numerical calculations, the light bullet propagation with Bessel profile is stable and the evolution we can see in Fig. 2.

As we see from evolution of the propagation of the two-dimensional few-circle optical pulses with Bessel profile in Bragg environment (Fig.2-3), the light bullet changes its configuration and we have an effect of the pulse spreading in time, as a result of dispersion effects.

The light bullet amplitude doesn't increase practically, and the form preserve it own view, subject to energy transfer in the lateral lobe. The solution for two-dimensional light bullet in Bragg environment with cross modulation stays localized, though doesn't change the spatial structure, as a result of cross dispersion. The combined action of effects of the pulse spreading, as a result of dispersion and nonlinearity leads to formation of complex structure in the of the pulse front, which stays located in limited space area 

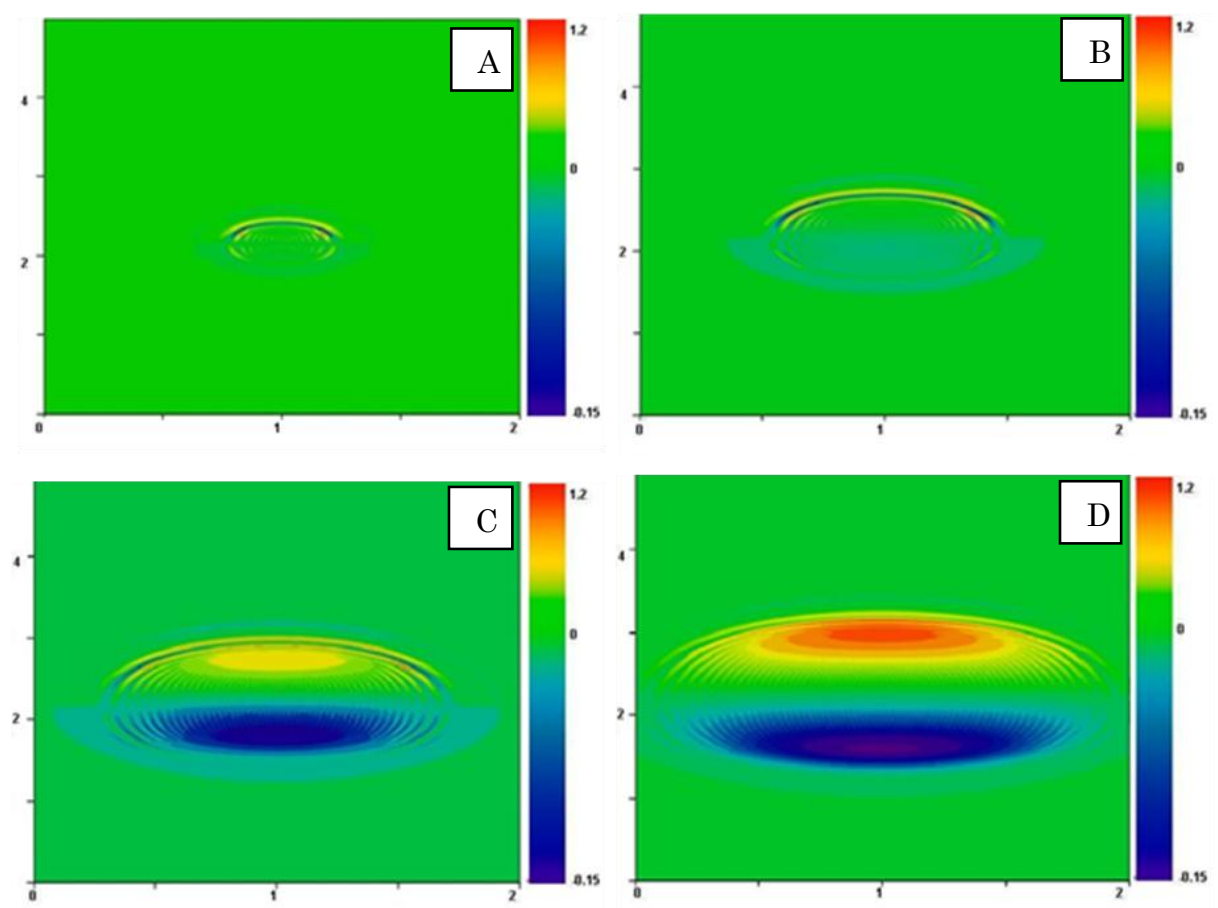

Fig. 2 - The light bullet propagation with Bessel profile in Bragg-environment with carbon nanotubes. Lattice period $2.5 \mathrm{mkm}$ and depth of modulation $\alpha=0,25$ in fixed time moment $T=2.5$ ps. a)T, b) $2 \mathrm{~T}, \mathrm{c}) 3 \mathrm{~T}, \mathrm{~d}) 4 \mathrm{~T}$
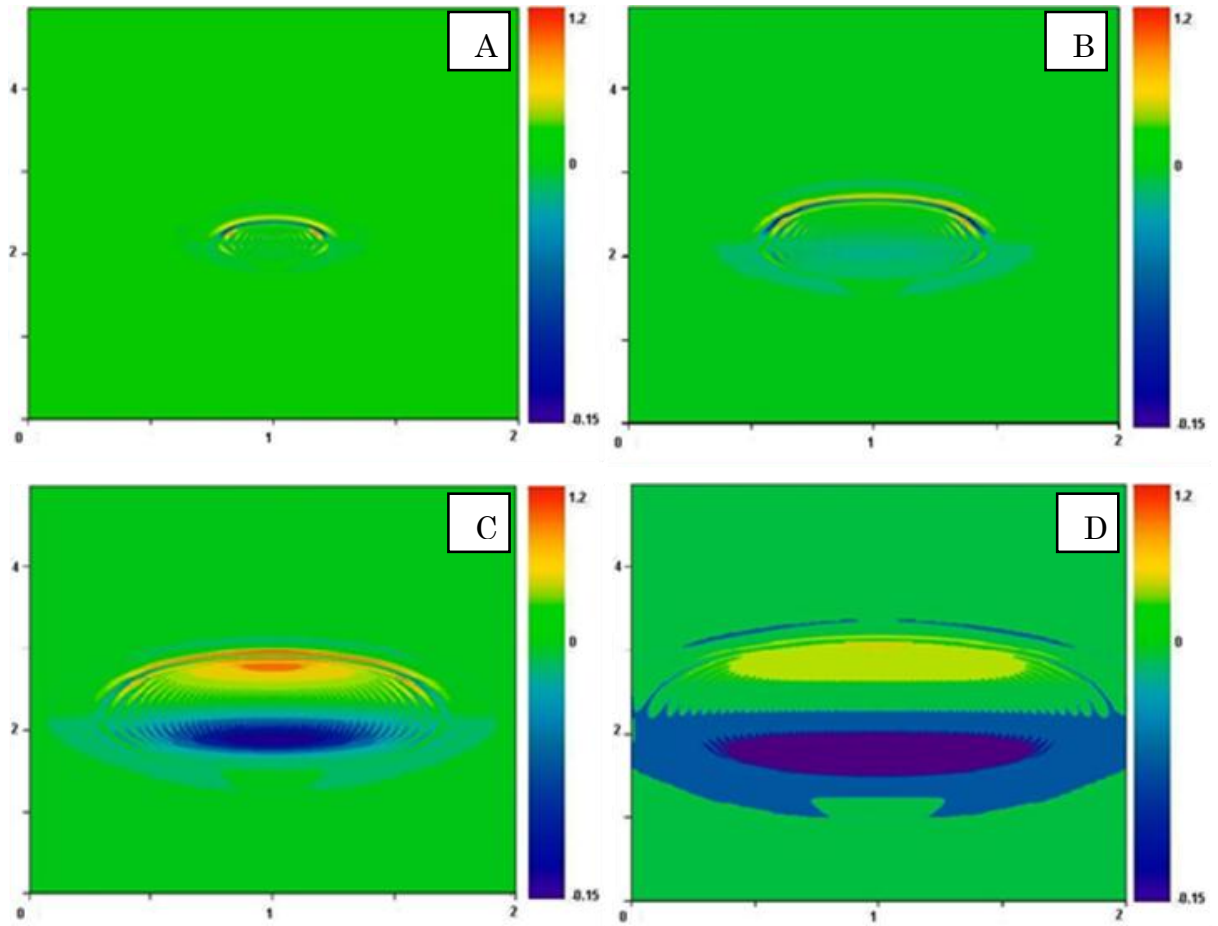

Fig. 3 - The light bullet propagation with Bessel profile in Bragg-environment with carbon nanotubes. Lattice period $5 \mathrm{mkm}$ and depth of modulation $\alpha=0,25$ in fixed time moment $T=2.5$ ps. a)T, b) $2 \mathrm{~T}$, c) $3 \mathrm{~T}$,d) $4 \mathrm{~T}$

The light bullet with Bessel profile is stable with respect to diffraction and the main role here play nonlinearity effects of environment.

We need to mark that the lattice brings to distortion of the pulse form as a result of wave interference, which test a partial reflection. The pulse slowdown is a result of that interference.

From Fig. 2-3. we can see that environment influences sizeable to the propagation velocity of the beam. When we increase a lattice period, the few-circle optical pulse propagates more quickly. Obviously, when a lattice period is infinite, as a result of absence of the interference process, the pulse will be propagate with maximal velocity. It was confirmed in our numerical calculations.

From practical viewpoint that result is impotent, 
because we can to manage the light bullets velocity by changing parameters of the Bragg environment. And with it, the light bullet propagation in Braggenvironment has a row of the important differences from case of environment with constant reflective index.

Perhaps, the main difference - the light bullets in Bragg environment have more compound cross structure, which tie together with stimulation of the internal oscillation mode of the light bullet interaction with heterogeneity of the environment refractive index. Earlier that effect we can see when we solved a task about interaction of the few-circle optical pulse with metal heterogeneity in the CNT environment [14].

\section{REFERENCES}

1. G. Fibich, B. Ilan, Opt. Lett. 29, 887 (2004).

2. P.M. Goorjian, Y. Silberberg, J. Opt. Soc. Am. B 14, 3253 (1997).

3. S.R.P. Pavani, A. Greengard, R. Piestun, Appl. Phys. Lett. 95, 545 (2009).

4. A.N.B. Spektor, J. Shamir, Appl. Opt. 47, A78 (2008).

5. N. Bozinovic, Ya. Yue, Yo. Ren, M. Tur, P. Kristenses, H. Huang, A.E. Willner, S. Ramachandran, Science, 340, 1545 (2013).

6. E. Abramochkin, S. Kotova, A. Korobtsov, N. Losevsky, A. Mayorova, M. Rakhmatulin, V. Volostnikov, Laser Phys. 16, $842(2006)$.

7. M.C. Roco, R.S. Williams, P. Alivisatos, Eds., (Russian edition), Nanotechnology Research Directions: Vision for Nanotechnology in the Next Decade (Moscow: Mir: 2002).

8. P.J.F. Harris, Carbon nanotubes and related structures: New materials for the 21st century (Cambridge: Cambridge

\section{CONCLUSION}

As a results of that research we can do the next resumes:

1. The few-circle optical pulses propagation in the environment with spatial modulate refractive index with carbon nanotubes is stable.

2. The Bragg environment in sizeable degree influences to pulse form and propagation velocity. The Bragg lattice period increase leads to increase of pulses velocity.

3. Light bullets in the Bragg-in environment have more composite structure thanks to stimulation of the internal oscillation mode of the bullet interaction with heterogeneity of the environment refractive index.

University Press: 2009).

9. Nanoelectromagnetics of low-dimensional structures. The Handbook of Nanotechnology: Nanometer Structure Theory, Modeling, and Simulation (Ed. by A. Lakhtakia), (Spain: SPIE Press: 2004).

10. M.B. Belonenko, E.V. Demushkinab, N.G. Lebedev, Phys. Solid State, 50, 383 (2008)

11. L.D. Landau, E.M. Livshits, Field Theory (Moscow: Fizmatlit: 1988) [in Russian].

12. S.J. Tans, M.H. Devoret, H. Dai, Nature, 386, 474 (1997).

13. N.S. Bakhvalov, Chislennyemetody (analiz, algebra, obyknovennyedifferentsialnye uravneniya) [Numerical methods (analysis, algebra, ordinary differential equations)] (Moscow: Nauka: 1975).

14. AS. Popov, M.B. Belonenko, N.G. Lebedev, Eur. Phys. J. D 65, 635 (2011). 